\title{
EFFECT OF MECHANICAL STRESSES ON THE MAGNETIC CHARACTERISTICS OF PIPELINE STEELS OF DIFFERENT CLASSES
}

\author{
A. N. Mushnikov ${ }^{1 *}$, S. Yu. Mitropolskaya ${ }^{2}$ \\ ${ }^{1}$ Institute of Engineering Science, Ural Branch of the Russian Academy of Sciences, 34 Komsomolskaya st., \\ Ekaterinburg, Russian Federation \\ ${ }^{2}$ Federal State Autonomous Educational Institution of Higher Professional Education "Ural Federal University named \\ after the first President of Russia B.N. Yeltsin”, 19 Mira str., Ekaterinburg, Russian Federation \\ *Corresponding author. E-mail: mushnikov@imach.uran.ru; address for correspondence: 34 Komsomolskaya st., \\ Ekaterinburg, Russian Federation. Tel.: +7(343)375 3587
}

The paper is concerned with the effect of applied macrostress on the magnetic characteristics of pipeline steels. Magnetic hysteresis parameters are examined as a function of tensile stress, tangential stress, and internal pressure. The study is performed on the hot-rolled 1010 steel alloyed with $\mathrm{Mn}$ and $\mathrm{Si}$, widely used in Russia, on the X42SS steel designed specially for operation in hydrogen-containing media and on the X70 steel produced by controlled rolling and intended for heavy-duty trunk pipelines. The results obtained are discussed in terms of the development of nondestructive physical techniques for the diagnosis of the current state of pipe material in course of operation.

Keywords: coercivity, remanent induction, maximum magnetic permeability, tensile stress, tangential stress, internal pressure loading, pipe steels.

DOI: $10.17804 / 2410-9908.2016 .4 .057-070$

\section{References}

1. Sultanov M.Kh., Makarov P.S., Zagidulin R.V., Muzhitsky V.F. Studying the possibility of monitoring the stress-strain state of the weld and the heat affected zone of welded joints in pipes by electromagnetic non-destructive instruments. Problemy sbora, podgotovki $i$ transporta nefti $i$ nefteproduktov, 2006, no. 66, pp. 164-179. (In Russian).

2. Gorkunov E.S., Ulyanov A.I. Magnitnye metody i pribory kontrolya kachestva izdeliy poroshkovoy metallurgii [Magnetic Methods and Equipment for Testing the Quality of Powder Metallurgy Products]. Ekaterinburg, Izd-vo UrO RAN Publ., 1996, 200 p. (In Russian).

3. Vonsovsky S.V., Shur Ya.S. Ferromagnetizm [Ferromagnetism]. M.; L., Gostekhteoretizdat, 1948, 816 p. (In Russian).

4. Makarov V.N., Biktashev T.K. Combined use of the longitudinal and transverse effects of magnetostriction in measuring the stresses in steel parts. Soviet Journal of Nondestructive TestingUSSR, 1981, vol. 17, iss. 5, pp. 378-382.

5. Barton I.R., Kuzenberger F.N. Estimating residual stresses in gas turbine engine parts by Barkhausen noise characteristics. Energeticheskie mashiny, 1975, no. 4, pp. 22-33. (In Russian).

6. Rautioaho R., Karjalanen L.P., Moilanen M. Stress response of Barkhausen noise and coercive force in 9Ni steel. Journal of Magnetism and Magnetic Materials, 1987, vol. 68, iss. 3, pp. 321-327. DOI: 10.1016/0304-8853(87)90008-4.

7. Stefanita C.G., Atherton D.L., Clapham L. Plastic versus elastic deformation effects on magnetic Barkhausen noise in steel. Acta Materialia, 2000, vol. 48, iss. 13, pp. 3545-3551. DOI: 10.1016/S1359-6454(00)00134-8.

8. Krause T.W., Clapham L., Pattantyus A., Atherton D.L. Investigation of the stressdependent magnetic easy axis in steel using magnetic Barkhausen noise. Journal of Applied Physics, 1996, vol. 79, iss. 8, pp. 4242-4252. DOI: 10.1063/1.361878. 
9. Inaguma T., Sakamoto H., Hasegawa M. Stress Dependence of Barkhausen Noise in Spheroidized Cementite Carbon Steel. IEEE Transactions on Magnetics, 2013, vol. 49, no. 4, pp. 1310-1317. DOI: 10.1109/TMAG.2012.2220856.

10. Zakharov V.A., Borovkova M.A., Babkin S.E. On the relation between coercive force and mechanical stresses in structural steels. In: Nerazrushayushchie phizicheskie metody i sredstva kontrolya materialov i izdeliy: tezisy dokladov konferentsii [Nondestructive Physical Methods and Means for Testing of Materials and Products: Abstracts of the Conference, Izhevsk, USSR, 1981]. Izhevsk, 1981, pp. 62-64. (In Russian).

11. Novikov V.F., Izosimov V.A. Influence of Elastic Stresses on Coercive Force. Fizika Metallov i Metallovedenie, 1984, vol. 58, iss. 2, pp. 275-281.

12. Musikhin S.A. On the feasibility of nondestructive evaluation of the stress state of pipelines in testing and operation. In: Neft i gaz Zapadnoy Sibiri. Problemy dobychi i transpotirovki: tezisy dokladov Vsesoyuznoy konferentsii [Oil and Gas of Western Siberia. Delivery and Transportation Problems: Abstracts of the All-Union Conference, Tyumen, USSR, 1985]. Tyumen, 1985, pp. 189-190. (In Russian).

13. Bolshakov V.N., Gorbash V.G., Olenovich T.V. Effect of Mechanical Stresses on Local Remanence. Izv. AN BSSR, ser. fiz. tekh. nauk, 1980, no. 1, pp. 109-112. (In Russian).

14. Gorkunov E.S., Novikov V.F., Nichiporuk A.P., Nassonov V.V., Kadrov A.V., Tatlybaeva I.N. Resistance of residual magnetization of heat-treated steel products to elastic deformations. Soviet Journal of Nondestructive Testing-USSR, 1991, vol. 27, no. 2, pp. 138-145.

15. Kuleev V.G., Bida G.V., Atangulova L.A. Feasibility of NDT of ferromagnetic steel structures based on measurements of residual magnetization as a function of elastic stress. Russian Journal of Nondestructive Testing, 2000, vol. 36, iss. 12, pp. 866-876. DOI: $10.1023 / \mathrm{A}: 1016714209905$.

16. Novikov V.F., Yatsenko T.A., Bakharev M.S. Coercive force of low-carbon steels as a function of uniaxial stress. Part I. Russian Journal of Nondestructive Testing, 2001, vol. 37, iss. 11, pp. 799-804. DOI: 10.1023/A:1015899320754.

17. Bida G.V. Magnetic method for estimating uniaxial compressive and tensile elastic stresses. Russian Journal of Nondestructive Testing, 2011, vol. 47, iss. 8, pp. 551-560. DOI: $10.1134 / \mathrm{S} 1061830911080043$.

18. Kostin V.N., Tsar'kova T.P., Loskutov V.E., Kostin K.V., Nichipuruk A.P., Lopatin V.V. Irreversible changes in the magnetization as indicators of stressed-strained state of ferromagnetic objects. Russian Journal of Nondestructive Testing, 2009, vol. 45, iss. 11, pp. 786-796. DOI: $10.1134 / \mathrm{S} 1061830909110059$.

19. Kostin K.V., Tsar'kova T.P., Nichipuruk A.P., Smorodinskii Ya.G. Measurement of the hysteresis characteristics of pipe steels under elastic and plastic tensile strain. Russian Journal of Nondestructive Testing, 2011, vol. 47, iss. 9, pp. 593-602. DOI: 10.1134/S1061830911090051.

20. Gorkunov E.S., Zadvorkin S.M., Veselov I.N., Mitropol'skaya S.Yu., Vichuzhanin D.I. Influence of Uniaxial Tension on Magnetic Characteristics of the 12 Gamma(sic) Pipe Steel Exposed to Hydrogen Sulfide. Russian Journal of Nondestructive Testing, 2008, vol. 44, iss. 8, pp. 566-573. DOI: 10.1134/S1061830908080093.

21. Gorkunov E.S., Zadvorkin S.M., Putilova E.A. Magnetic estimation of stresses applied to a two-layer steel $\mathrm{C}(\mathrm{T}) 3$-steel $08 \mathrm{X} 18 \mathrm{H} 10 \mathrm{~T}$ composite material during elastoplastic deformation by uniaxial tension. Russian Journal of Nondestructive Testing, 2012, vol. 48, iss. 8, pp. 495-504. DOI: $10.1134 / \mathrm{S} 1061830912080050$.

22. Ren Shang-kun, Ou Yang-chun, Fu Ren-zhen, Fu Yue-wen. Studies on stress-magnetism coupling effect for 35 steel components. Insight-Non-Destructive Testing \& Condition Monitoring, 2010, vol. 52, iss. 6, pp. 305-309(5). DOI: http://dx.doi.org/10.1784/insi.2010.52.6.305.

23. Nichipuruk A.P., Stashkov A.N., Kostin V.N., Korkh M.K. Possibilities of magnetic inspection of plastic deformations preceding failures of low-carbon steels constructions. Russian Journal of Nondestructive Testing, 2009, vol. 45, iss. 9, pp. 616-622. DOI: 10.1134/S1061830909090034.

Mushnikov A. N. et al. / Effect of mechanical stresses on the magnetic characteristics of pipeline steels of 
24. Gorkunov E.S., Mitropolskaya S.Yu., Groznaya E.M., Mushnikov A.N., Osintseva A.L., Tueva E.A. Effect of Elasto-Plastic Loading on the Magnetic Characteristics of Steel 20 Hardened with Gas Case-Hardening. Russian Journal of Nondestructive Testing, 2011, vol. 47, iss. 4, pp. 221-231. DOI: 10.1134/S106183091104005X.

25. Dunaev F.E. Protsessy peremagnichivaniya ferromagnetikov [Processes of Magnetization Reversal in Ferromagnets]. Sverdlovsk, UrGU Publ., 1979, 89 p. (In Russian).

26. Gorkunov E.S., Zadvorkin S.M., Mushnikov A.N., Smirnov S.V., Yakushenko E.I. Effect of mechanical stresses on the magnetic characteristics of pipe steel. Journal of Applied Mechanics and Technical Physics, 2014, vol. 55, iss. 3, pp. 530-538. DOI: 10.1134/S002189441403016X.

27. Sablik M.J., Jiles D.C. Modeling the Effects of Torsional Stress on Hysteretic Magnetization. IEEE Transactions on Magnetics, 1999, vol. 35, no. 1, part 2, pp. 498-504. DOI: $10.1109 / 20.737472$. 
Подана в журнал: 16.08 .2016

УДК 537.622

DOI: $10.17804 / 2410-9908.2016 .4 .057-070$

\title{
ВЛИЯНИЕ МЕХАНИЧЕСКОГО НАГРУЖЕНИЯ НА МАГНИТНЫЕ ХАРАКТЕРИСТИКИ ТРУБНЫХ СТАЛЕЙ РАЗНЫХ КЛАССОВ
}

\author{
А. Н. Мушников ${ }^{1}$, С. Ю. Митропольская ${ }^{2}$ \\ ${ }^{1}$ Федеральное государственное бюджетное учреждение науки Институт машиноведения Уральского \\ отделения Российской академии наук, ул. Комсомольская, 34, Екатеринбург, Российская Федерация \\ ${ }^{2}$ Федеральное государственное автономное образовательное учреждение выстего образования \\ «Уральский федеральный университет имени первого Президента России Б.Н. Ельцина», ул. Мира, 19, \\ Екатеринбург, Российская Федераиия
}

*Ответственный автор. Электронная почта: mushnikov@imach.uran.ru; Адрес для переписки: Россия, Екатеринбург, ул. Комсомольская, 34; Телефон: +7 (343) 375-35-87

Дан обзор существующих представлений о влиянии приложенных макронапряжений на магнитные свойства сталей. Рассмотрено влияние напряжений, возникающих при растяжении, кручении и нагружении внутренним давлением, на гистерезисные магнитные характеристики трубных сталей трех классов. Исследовали образцы широко применяемой горячекатаной стали 09Г2С, устойчивой к сероводородному воздействию стали 12ГБ и стали класса X70, полученной методом контролируемой прокатки. Обсуждено применение магнитных методов контроля для оценки технического состояния трубопроводов в рабочих условиях их эксплуатации.

Ключевые слова: коэричитинная сила, остаточная индукиия, максимальная магнитная проницаемость, растягивающие напряжения, касательные напряжения, внутреннее давление, трубные стали.

\section{1. Введение}

Напряженно-деформированное состояние трубопроводов является одной из важнейших характеристик, определяющих возможность их надежной эксплуатации. В процессе эксплуатации они подвергаются действию переменных во времени механических нагрузок, которые могут стать причиной усталостных изменений структуры металла и накопления микродефектов, а следовательно, образования макродефектов, зарождения трещин и разрушения изделия. Все это может привести не только к материальным потерям, но и в определенных ситуациях к негативному влиянию на окружающую среду и гибели людей. Зная о действующих напряжениях, можно принять превентивные меры: усилить опасный участок трубопровода, либо снять напряжения внесением изменений в конструкцию.

В последние годы большой интерес проявляется к магнитным методам контроля напряженно-деформированного состояния стальных конструкций, которые основаны на корреляции между магнитными характеристиками и механическими параметрами металлов. Разработанные методы и технические средства измерения механических напряжений металла по магнитным диагностическим параметрам обладают рядом преимуществ, важных для ранней диагностики напряженно-деформированного состояния элементов конструкций: неразрушающее действие операции контроля, достаточно высокая производительность контроля в полевых условиях, возможность дистанционного контроля [1].

Магнитные методы контроля напряжений в сталях лучше всего применимы для измерения одноосных напряжений. Эти методы отличаются экспрессностью получения информаMushnikov A. N. et al. / Effect of mechanical stresses on the magnetic characteristics of pipeline steels of 
ции и удобством проведения измерений [2]. Однако магнитные методы требуют тщательного исследования влияния на результаты измерений различных факторов, таких как химический состав, структурное состояние, механическая предыстория.

Под действием механических напряжений изменяются параметры петли магнитного гистерезиса и кривой намагничивания [3]. Изменениям подвержены такие характеристики, как коэрцитивная сила, остаточная намагниченность, магнитная проницаемость (начальная, максимальная, дифференциальная), магнитострикция, структура шумов Баркгаузена и другие. Эти характеристики можно использовать для оценки напряженно-деформированного состояния в тех случаях, когда изменения магнитных характеристик материала имеют однозначную связь с изменением приложенных напряжений.

Начало практической реализации этой идеи относится к 70-м годам прошлого века. Так, например, магнитострикционный метод был применен в работе [4]. При помощи тензодатчиков проводились измерения продольной и поперечной магнитострикции. Было установлено, что с ростом растягивающих напряжений отношение величин продольной магнитострикции к поперечной уменьшается. Несмотря на то, что в лабораторных условиях предложенный метод обладает высокой чувствительностью, он сложен в практической реализации, так как требует предварительную оклейку контролируемого объекта тензодатчиками в ненагруженном состоянии, а также защиту датчиков от повреждения. Точность метода чувствительна к качеству приклейки тензодатчиков, изменениям температуры окружающей среды и структурных свойств металла. Примерно в то же время появились работы, в которых для определения механических напряжений был использован эффект Баркгаузена [5-6]. Исследования показали, что параметры скачков Баркгаузена являются высокочувствительными к упругим напряжениям. В настоящее время достаточно большое количество работ посвящено использованию магнитных шумов Баркгаузена для оценки напряженно-деформированного состояния. Например, в работе [7] проведено сравнение влияния упругих и пластических деформаций на линейные и угловые шумы Баркгаузена. Установлено, что упругие деформации значительно влияют на магнитную анизотропию в образце, но изменение изотропного сигнала очень мало. Пластическая деформация оказывает меньший, но противоположный эффект - значительно меняется изотропный сигнал, но изменения анизотропии практически отсутствуют. В работе [8] на образцах трубной стали исследовалось влияние направления действующих напряжений на зависимость магнитных шумов Баргаузена от величины напряжений. Наибольший сигнал был получен при растяжении перпендикулярно оси легкого намагничивания, и при сжатии вдоль оси легкого намагничивания. В работе [9] изучено влияние механических напряжений на шумы Баргаузена для цементированных сталей. На основании измерений описана модель объясняющая, почему возрастает количество $90^{\circ}$ доменов в результате перемещения $180^{\circ}$ доменов.

Однако ограниченность метода магнитных шумов Баркгаузена состоит в том, что он позволяет получать информацию только от поверхностных слоев металла, свойства которых могут отличаться от свойств объекта в целом. Кроме того, эффект существенно зависит от качества подготовки поверхности.

Еще один метод определения упругих напряжений основан на корреляции одноосных напряжений с характеристиками петли магнитного гистерезиса и кривой намагничивания (коэрцитивной силой, остаточной намагниченностью, магнитной проницаемостью или восприимчивостью). Зачастую из магнитных параметров выбирают коэрцитивную силу, в связи с относительной легкостью ее измерения независимо от геометрии объекта исследования. В работе [10] были представлены результаты изучения влияния упругих и пластических деформаций на коэрцитивную силу сталей 10 и 45, которые показали возможность использования коэрцитивной силы в качестве параметра контроля одноосных напряжений. В работе [11] экспериментально изучена зависимость от величины растягивающих напряжений коэрцитивной силы, измеряемой вдоль и поперек их приложения сплава железо-кобальт, у которого константы магнитострикции $\lambda_{100}$ и $\lambda_{111}$ положительны. В [12] приведены результаты из-

Mushnikov A. N. et al. / Effect of mechanical stresses on the magnetic characteristics of pipeline steels of 
мерений коэрцитивной силы, как функции напряжений на сталях 3, 20 и 45. Показано, что зависимость коэрцитивной силы от напряжений может существенно различаться для разных сталей.

Другим параметром петли магнитного гистерезиса, имеющим хорошую корреляцию с механическими напряжениями, является остаточная намагниченность. В [14] изучена устойчивость остаточной намагниченности ряда конструкционных сталей с содержанием углерода 0,3-0,4 \% к воздействию растягивающих, сжимающих, крутящих и знакопеременных циклических упругих деформаций. Монотонные изменения остаточной намагниченности, наблюдаемые при возрастании действующих на ферромагнетик упругих напряжений, позволяют оценить их величину.

Для многих сталей зависимость коэрцитивной силы и других гистерезисных свойств от упругих растягивающих напряжений является немонотонной, что исключает возможность контроля напряженно-деформированного состояния по одному параметру. Для оценки напряжений в этом случае обычно используют несколько магнитных характеристик, полученных при перемагничивании исследуемого объекта в разных направлениях. В [15] проблема сравнительно малых изменений и немонотонности зависимостей отдельных магнитных параметров от напряжений при растяжении решена путем измерения остаточной намагниченности в трех взаимно-перпендикулярных направлениях: в направлении нагружения, перпендикулярно направлению нагружения и под углом $45^{\circ}$ к нему. В работе [16] были получены корреляционные зависимости, описывающие изменение коэрцитивной силы, измеряемой вдоль и поперек направления приложения растягивающей нагрузки, и осуществлена проверка полученных соотношений на нескольких малоуглеродистых сталях. Однако данный метод не применим в случаях, когда схема нагружения отлична от одноосной. В [17] показано, как по коэрцитивной силе, релаксационной намагниченности и релаксационной магнитной восприимчивости в многофакторной модели можно контролировать величину одноосных напряжений растяжения и сжатия при упругом нагружении.

Чувствительность магнитных характеристик к приложенным напряжениям может различаться для разных сталей. Как правило, в работах рассматривают комплекс магнитных характеристик $[14-16,19-24]$ и делают выводы о возможности использования той или иной характеристики для контроля действующих механических напряжений с учетом особенностей объекта контроля. Цель данной работы - изучение влияния механического нагружения на магнитные характеристики трубных сталей трех классов: горячекатаной стали 09Г2С, устойчивой к сероводородному воздействию стали 12 ГБ и стали класса X70, полученной методом контролируемой прокатки.

\section{2. Материал и методика исследования}

В качестве материала исследования были выбраны трубные стали трех различных классов, из числа наиболее широко применяемых в настоящее время в нашей стране. Это горячекатаная сталь марки 09Г2С, в течение многих лет хорошо зарекомендовавшая себя в регионах холодного климата, а также две стали нового поколения - микролегированная ниобием сталь 12 ББ, предназначенная для эксплуатации на месторождениях кислого природного газа, и сталь контролируемой прокатки класса прочности X70, микролегированная молибденом, ванадием и ниобием. Маркировка X70 по стандартам Американского нефтяного института API 5LX и API 5LS указывает на предел текучести в единицах Ksi, что соответствует приблизительно $485 \mathrm{MПа} \mathrm{(1} \mathrm{Ksi}=1000$ psi = 6,894 МПа). Данные табл. 1 позволяют на качественном уровне сравнить структуру исследованных сталей.

Для исследования сталей 09Г2С и 12ГБ были выбраны фрагменты бесшовных труб, а для исследования стали X70 - фрагмент спиральношовной трубы большого диаметра. Пятикратные цилиндрические образцы по ГОСТ 1497-84 вырезали из фрагментов труб вдоль направления прокатки, а для исследования стали X70 - вдоль и поперек направления прокат-

Mushnikov A. N. et al. / Effect of mechanical stresses on the magnetic characteristics of pipeline steels of 
ки. Кроме того, для изучения влияния внутреннего давления были изготовлены полые цилиндрические образцы из трубной стали 09Г2С. Внешний радиус рабочей части таких образцов равен 5,5 мм, ее внутренний радиус- 4,5 мм, длина рабочей части равна 60 мм.

Таблица 1 - Основные структурные характеристики, технология получения и назначение исследованных трубных сталей

\begin{tabular}{|c|c|c|c|}
\hline Марка & $09 \Gamma 2 \mathrm{C}$ & $12 Г Б$ & $\mathrm{X70}$ \\
\hline Назначение & $\begin{array}{c}\text { Магистральные и } \\
\text { немагистральные } \\
\text { трубопроводы в } \\
\text { условиях холодно- } \\
\text { го климата }\end{array}$ & $\begin{array}{c}\text { Газосборные сети } \\
\text { месторождений с } \\
\text { высоким содержа- } \\
\text { нием } \mathrm{H}_{2} \mathrm{~S} \text { и } \mathrm{CO}_{2}\end{array}$ & $\begin{array}{c}\text { Магистральные трубопроводы } \\
\text { повышенного давления в усло- } \\
\text { виях холодного климата }\end{array}$ \\
\hline $\begin{array}{l}\text { Технология } \\
\text { получения }\end{array}$ & Горячая прокатка & $\begin{array}{c}\text { Горячая прокатка }+ \\
\text { нормализация }\end{array}$ & Контролируемая прокатка \\
\hline $\begin{array}{c}\text { Основные } \\
\text { структурные } \\
\text { составляю- } \\
\text { щие }\end{array}$ & феррит + перлит &  & $\begin{array}{c}{ }^{\sigma} \longleftarrow \text { } \\
\text { феррит + бейнито- } \\
\text { мартенситная смесь }+ \\
(\mathrm{V}, \mathrm{Nb})(\mathrm{CN}) \text { по субграницам }\end{array}$ \\
\hline $\begin{array}{c}\text { Структурная } \\
\text { неоднород- } \\
\text { ность }\end{array}$ & $\begin{array}{c}\text { Структура изо- } \\
\text { тропная }\end{array}$ & $\begin{array}{c}\text { Структура изотроп- } \\
\text { ная }\end{array}$ & $\begin{array}{c}\text { Структура анизотропная отно- } \\
\text { сительно оси прокатки }\end{array}$ \\
\hline Размер зерна & балл 7-8 & балл 8-9 & $\begin{array}{c}\text { балл 10-11 - вдоль, балл 11-12 - } \\
\text { поперек оси прокатки }\end{array}$ \\
\hline Доля перлита & $\sim 10 \%$ & $\sim 15 \%$ & $<5 \%$ \\
\hline
\end{tabular}

Таблица 2 - Механические и магнитные характеристики исследованных трубных сталей

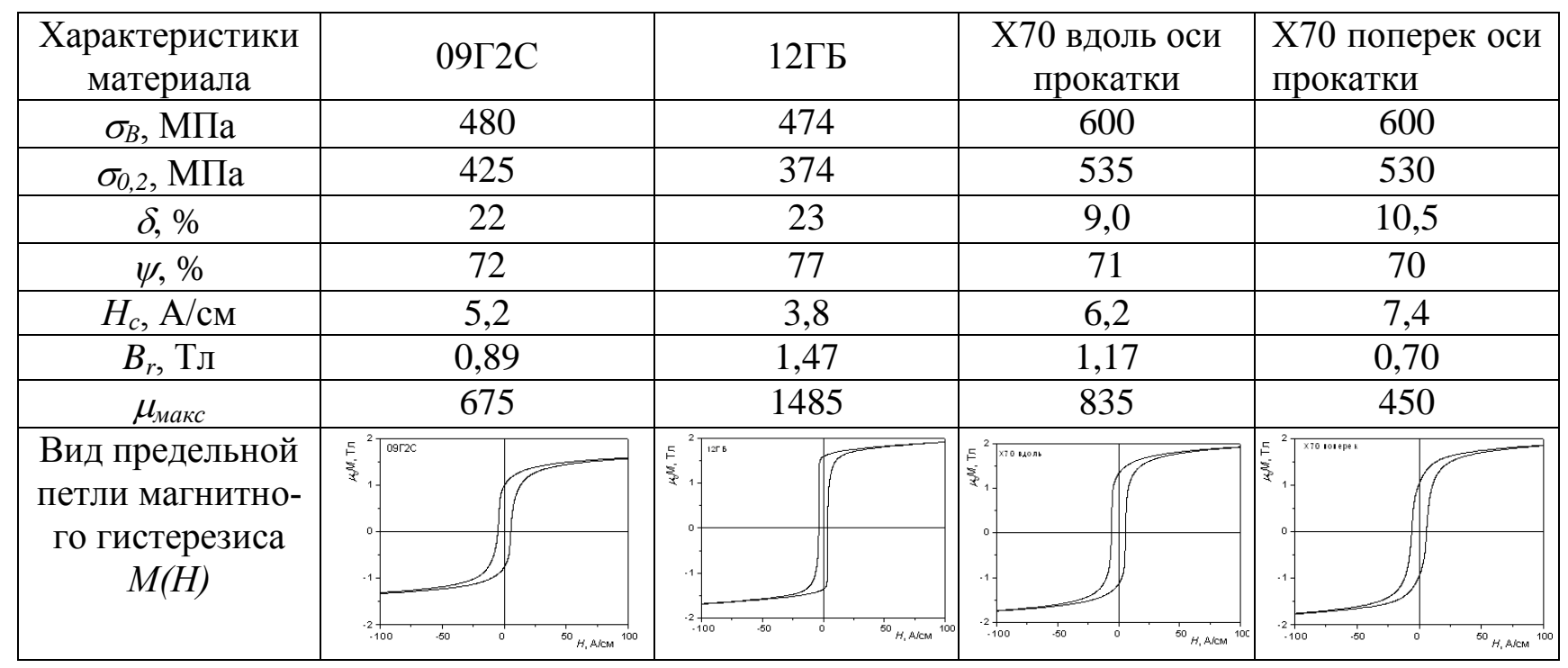

Mushnikov A. N. et al. / Effect of mechanical stresses on the magnetic characteristics of pipeline steels of 
Испытания проводили на установке, с помощью которой можно одновременно осуществлять одноосное растяжение (сжатие), кручение, создавать гидростатическое давление во внутренней полости образца [26], а также проводить измерения магнитных характеристик. При испытаниях на кручение полые образцы имеют меньшую неоднородность сдвиговых деформаций по сечению, по сравнению со сплошными цилиндрами.

Механические характеристики исследованных сталей были определены предварительно на испытательной машине Instron (табл. 2). Магнитные измерения при упругом деформировании проводили по схеме пермеаметра. Коэрцитивную силу $H_{C}$ и остаточную индукцию $B_{\mathrm{r}}$ определяли на предельной петле магнитного гистерезиса с напряженностью маг-

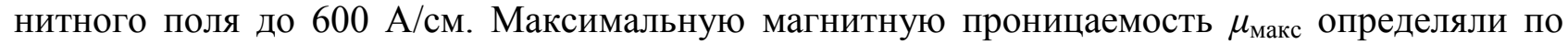
кривой намагничивания. Магнитные характеристики определяли в исходном состоянии без нагрузки (табл. 2) и под действием приложенных растягивающих, касательных напряжений и внутреннего гидростатического давления. Перед началом каждого магнитного измерения и по его окончанию образец размагничивали.

\section{3. Результаты и обсуждение}

\section{1 Действие растягивающих напряжений}

На рис. 1 приведены диаграммы изменения коэрцитивной силы $H_{C}$, остаточной ин-


ствием приложенных растягивающих напряжений.

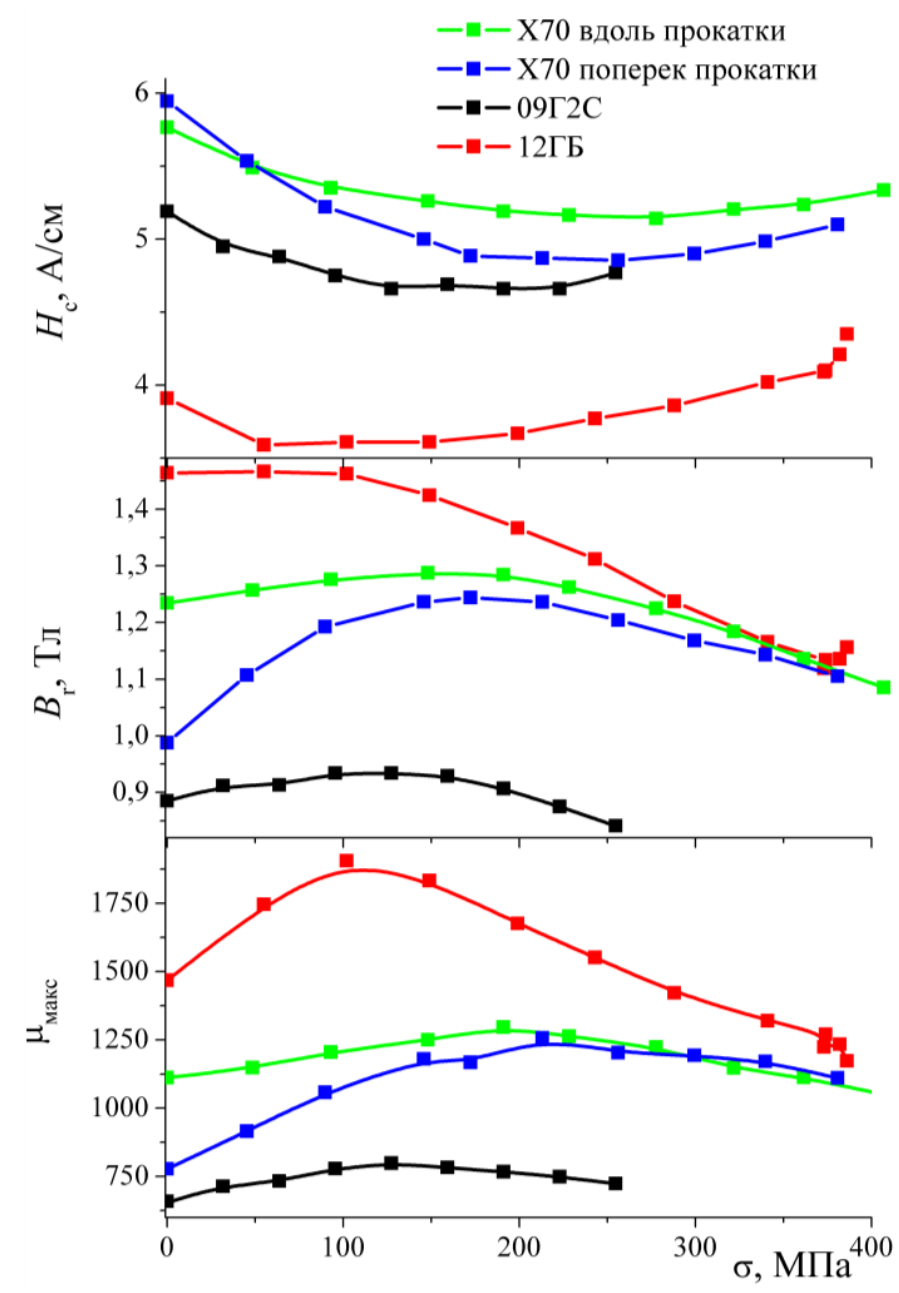

Рис. 1. Влияние растягивающих напряжений на магнитные характеристики исследованных сталей

Mushnikov A. N. et al. / Effect of mechanical stresses on the magnetic characteristics of pipeline steels of 
Для всех испытанных образцов наблюдается немонотонный двухстадийный ход кривых. Так, сопоставление кривых $H_{C}$ (рис. $1 a$ ) показывает, что характер изменения коэрцитивной силы при силовом нагружении всех испытанных образцов принципиально подобен: снижение $H_{C}$ в упругой области за счет положительной магнитострикции, более выраженное поперек прокатки, сменяется при переходе к пластическому нагружению умеренным ростом.

Согласно [25] на материалах с положительной магнитострикцией при воздействии растягивающих напряжений $(\lambda \sigma>0)$ формируется магнитная текстура с преимущественной ориентацией магнитных моментов доменов вдоль направления приложения нагрузки. Поэтому с увеличением растягивающих напряжений $(\sigma>0)$ магнитные характеристики трубных сталей ведут себя немонотонно: коэрцитивная сила начинает возрастать, а остаточная индукция уменьшаться под действием растягивающих напряжений: для стали 09Г2С - более 150 МПа, для стали 12ГБ - более 100 МПа, а для стали Х70 - свыше 250 МПа. Это связано с тем, что полевая зависимость продольной магнитострикции в случае действия растягивающих напряжений переходит в отрицательную область в меньших полях.

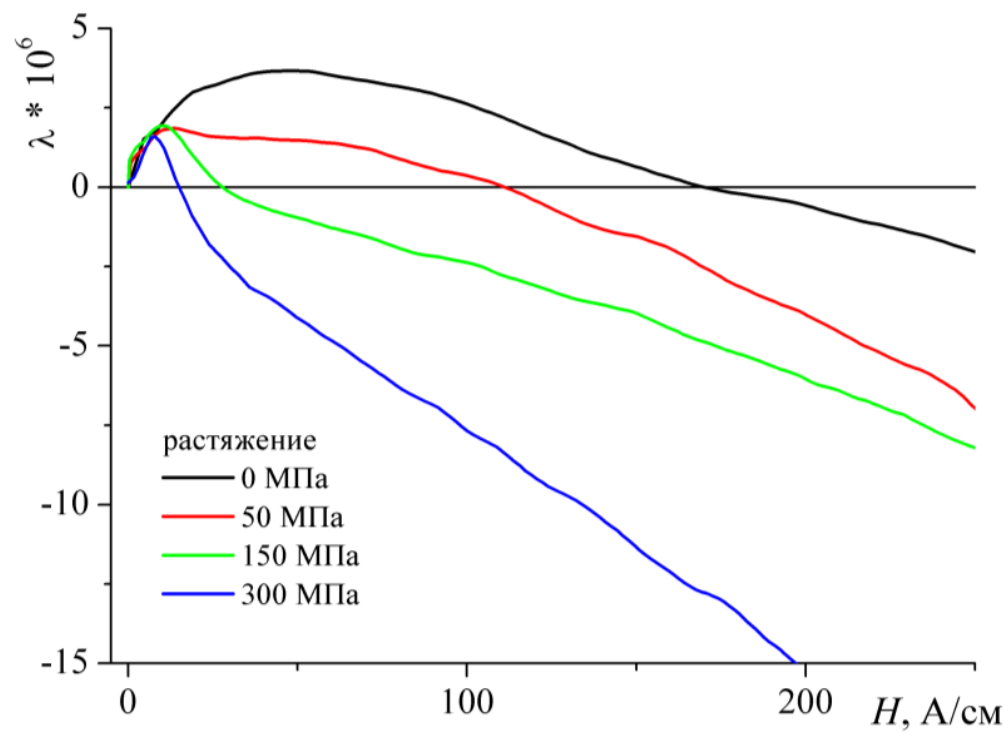

Рис. 2. Влияние растягивающих напряжений на полевые зависимости продольной магнитостриции стали 09Г2С

Согласно рис. 2 с увеличением внешнего магнитного поля продольная магнитострикция сначала принимает положительные значения, а затем, в полях выше 170 A/см, становится отрицательной. При растягивающих напряжениях 150 МПа поле $H$, в котором $\lambda$ становится отрицательной, составляет $28 \mathrm{~A} / \mathrm{cm}$, что в 6 раз меньше относительно ненагруженного состояния. А при напряжениях 300 МПа поле смены знака магнитострикции в 12 раз меньше, чем в ненагруженном состоянии [26]. Вследствие изменения знака магнитострикции реакция всех магнитных характеристик на напряжения меняется на противоположную.

Особое внимание обращают на себя магнитные характеристики образцов стали X70, вырезанных в разных направлениях относительно оси прокатки. Из кривых, представленных на рис. 1, видно, что с увеличением растягивающих напряжений до 200 МПа наиболее интенсивно меняются магнитные характеристики образцов, вырезанных поперек направления прокатки. Понятно, что намагничивание образцов, вырезанных вдоль направления прокатки, и без нагрузки проходило относительно легко, а возникновение положительного магнитоупругого эффекта еще несколько упрощает этот процесс.

Важно отметить, что при напряжениях около 250 МПа абсолютные значения остаточ-

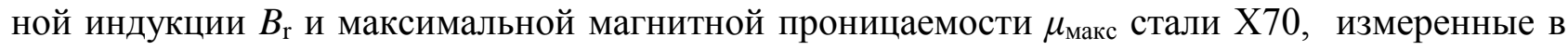
различных направлениях по отношению к оси прокатки, выравниваются между собой и при

Mushnikov A. N. et al. / Effect of mechanical stresses on the magnetic characteristics of pipeline steels of 
дальнейшем увеличении нагрузки не зависят от направления вырезки образцов. Приведенные на рис. 1 кривые для стали X70 свидетельствуют, что под действием растягивающих напряжений выше 200...250 МПа влияние структурной анизотропии контролируемой прокатки на магнитные свойства трубной стали перекрывается более сильным воздействием магнитной текстуры напряжений.

\section{2 Действие касательных напряжений}

На рис. 3 показано, как влияют приложенные касательные напряжения на магнитные характеристики сталей 09Г2С и Х70.

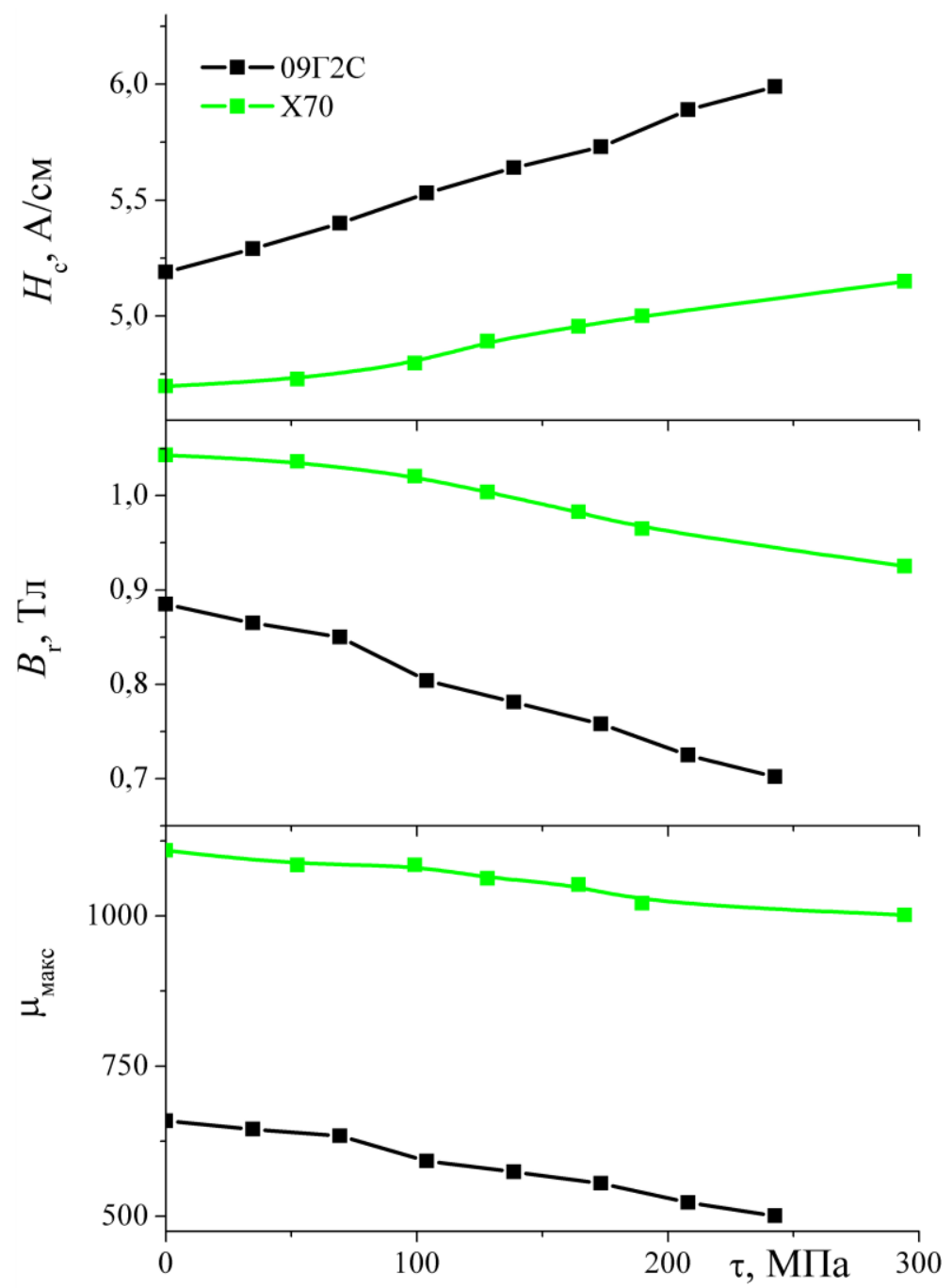

Рис. 3. Влияние касательных напряжений на магнитные характеристики исследованных сталей

Касательные напряжения при кручении $\tau$ в каждой точке эквивалентны паре нормальных взаимно-перпендикулярных растягивающих и сжимающих напряжений, равных по модулю $\tau$, и действующих в касательной к цилиндру плоскости под углом $45^{\circ}$ к оси образца. Поэтому под действием касательных напряжений вектора магнитных моментов доменов стремятся выстроиться под углом $45^{\circ}$ к оси образца (вдоль направления растягивающих напряжений), а после намагничивания в магнитном поле $H$ вектор результирующей намагниченности располагается под некоторым углом $\alpha$ к направлению приложенного магнитного поля [27]. Угол $\alpha$ находится в интервале от 0 до $45^{\circ}$, в зависимости от величины касательных напряжений и прикладываемого магнитного поля $H$. Очевидно, в таком случае проекция век-

Mushnikov A. N. et al. / Effect of mechanical stresses on the magnetic characteristics of pipeline steels of 
тора результирующей намагниченности на ось $z$ образца $\left(\mathrm{pr}_{z} M\right)$ будет меньше величины результирующей намагниченности в ненагруженном состоянии, т.е. процессы намагничивания будут проходить труднее.

Из рис. 3 видно, что рост касательных напряжений в интервале от 0 до 300 МПа приводит к линейному росту коэрцитивной силы, уменьшению остаточной индукции и максимальной магнитной проницаемости исследованных сталей. При линейной аппроксимации коэффициент детерминации превосходит 0,98. При повышении касательных напряжений до 300 МПа изменения всех магнитных характеристик составляют не менее 12-15\%. Данный факт позволяет использовать любую из рассмотренных характеристик для контроля упругих напряжений при кручении.

\section{3 Действие внутреннего гидростатического давления}

Внутреннее гидростатическое давление вызывает растягивающие окружные напряжения $\sigma_{\theta}$ и сжимающие радиальные напряжения $\sigma_{r}$, причем $\left|\sigma_{\theta}\right| \gg\left|\sigma_{r}\right|$. Окружные и радиальные напряжения расположены перпендикулярно направлению намагничивания. Под действием сжимающих напряжений $\sigma_{r}$ вектора магнитных моментов доменов стремятся повернуться в плоскость, касательную к цилиндру, а под действием растягивающих $\sigma_{\theta}-$ вдоль линии действия этих напряжений. Так как наибольшими (по модулю) являются растягивающие окружные напряжения, то под действием давления результирующая намагниченность должна уменьшиться, т.е. процессы намагничивания будут проходить труднее, чем в ненагруженном состоянии. Под действием внутреннего давления коэрцитивная сила увеличивается, а остаточная индукция уменьшается (рис. 4).

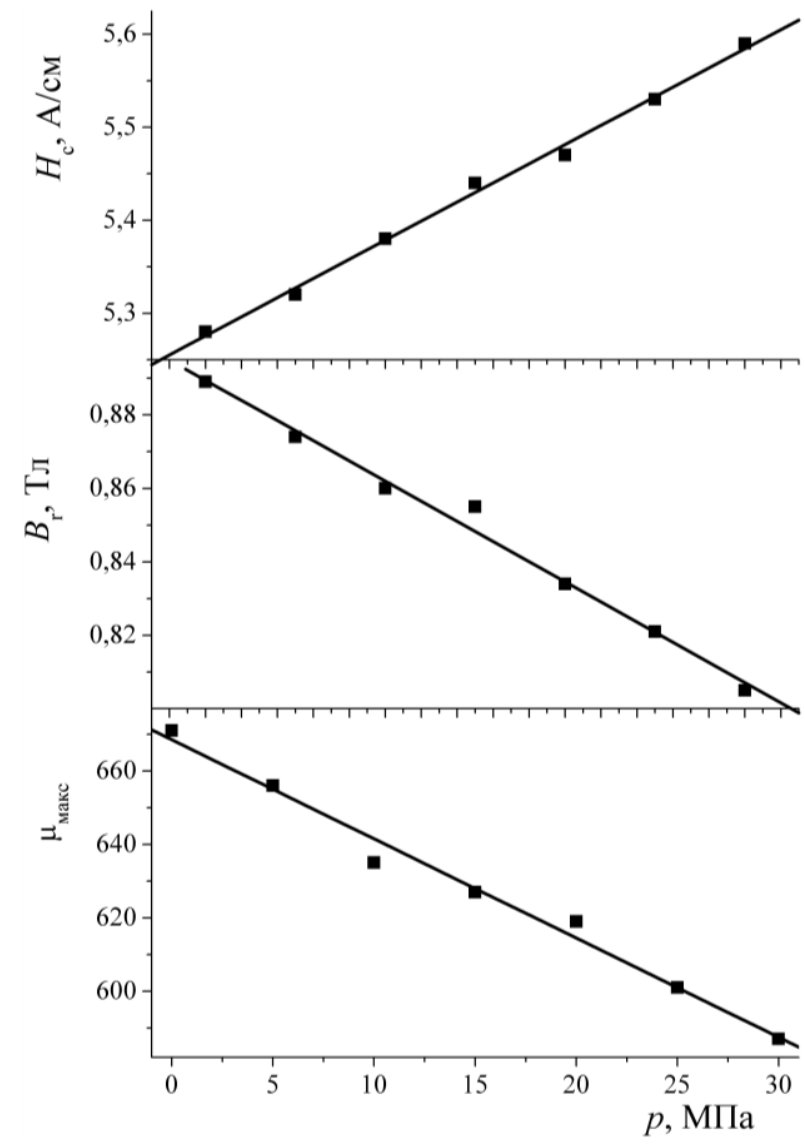

Рис. 4. Влияние внутреннего давления на магнитные характеристики стали 09Г2С

Mushnikov A. N. et al. / Effect of mechanical stresses on the magnetic characteristics of pipeline steels of 
При повышении внутреннего давления до 30 МПа изменения характеристик составляют $\sim 5 \%$. Количественно окружные напряжения влияют на коэрцитивную силу примерно в полтора - два раза слабее, чем напряжения одноосного сжатия.

\section{4. Заключение}

Показано влияние приложенных растягивающих и касательных макронапряжений, а также внутреннего гидростатического давления на магнитные свойства трубных сталей трех различных классов. На всех рассмотренных сталях наблюдается немонотонное изменение магнитных характеристик под действием приложенных растягивающих напряжений в интервале упругих напряжений от 0 до 400 МПа. В то же время зависимость исследованных магнитных характеристик от приложенных касательных напряжений в интервале от 0 до 300 МПа и от внутреннего гидростатического давления в интервале от 0 до 30 МПа носит линейный характер и указывает на высокую перспективность магнитного метода для оценки изменений напряженно-деформированного состояния трубопроводов.

\section{Благодарность}

Работа выполнена при частичной поддержке гранта РФФИ №16-38-00598 мол_а. При выполнении исследований использовалось оборудование ЦКП «Пластометрия».

\section{Литература}

1. Исследование возможности контроля напряженно-деформированного состояния сварного шва и околошовной зоны трубы с помощью электромагнитных приборов неразрушающего контроля / М. Х. Султанов, П. С. Макаров, Р. В. Загидулин, В. Ф. Мужицкий // Проблемы сбора, подготовки и транспорта нефти и нефтепродуктов. 2006. - № 66. - С. 164-179.

2. Горкунов Э. С., Ульянов А. И. Магнитные методы и приборы контроля качества изделий порошковой металлургии. - Екатеринбург : Изд-во УрО РАН, 1996. - 200 с.

3. Вонсовский С. В., Шур Я. С. Ферромагнетизм. - М.; Л. : Гостехтеоретиздат, 1948. $816 \mathrm{c}$.

4. Makarov V. N., Biktashev T. K. Combined use of the longitudinal and transverse effects of magnetostriction in measuring the stresses in steel parts // Soviet Journal of Nondestructive Testing - USSR. - 1981. - Vol. 17, iss. 5. - P. 378-382.

5. Бартон И. Р., Кузенбергер Ф. Н. Оценка остаточных напряжений в деталях газотурбинных двигателей по характеру Баркгаузеновского шума. // Энергетические машины. 1975. - № 4. - C. 22-33.

6. Rautioaho R., Karjalanen P., Moilanen M. Stress response of Barkhausen noise and coercive force in 9Ni steel // Journal of Magnetism and Magnetic Materials. - 1987. - Vol. 68, iss. 3. P. 321-327. - DOI: 10.1016/0304-8853(87)90008-4.

7. Stefanita C. G., Atherton D. L., Clapham L. Plastic versus elastic deformation effects on magnetic Barkhausen noise in steel // Acta Materialia. - 2000. - Vol. 48, iss. 13. - P. 3545-3551. DOI: $10.1016 / \mathrm{S} 1359-6454(00) 00134-8$.

8. Investigation of the stress-dependent magnetic easy axis in steel using magnetic Barkhausen noise / T. W. Krause, L. Clapham, A. Pattantyus, D. L. Atherton // Journal of Applied Physics. 1996. - Vol. 79, iss. 8. - P. 4242-4252. - DOI: 10.1063/1.361878.

9. Inaguma T., Sakamoto H., Hasegawa M. Stress Dependence of Barkhausen Noise in Spheroidized Cementite Carbon Steel // IEEE Transactions on Magnetics. - 2013. - Vol. 49, no. 4. P. 1310-1317. - DOI: 10.1109/TMAG.2012.2220856.

10. Захаров В. А., Боровкова М. А., Бабкин С. Э. О связи коэрцитивной силы с механическими напряжениями в конструкционных сталях // Неразрушающие физические методы и средства контроля материалов и изделий : тезисы докладов. - Ижевск, 1981. - С. 62-64.

Mushnikov A. N. et al. / Effect of mechanical stresses on the magnetic characteristics of pipeline steels of 
11. Novikov V. F., Izosimov V. A. Influence of Elastic Stresses on Coercive Force // Fizika Metallov i Metallovedenie. - 1984. - Vol. 58, iss. 2. - P. 275-281.

12. Мусихин С. А. О возможности неразрушающего контроля напряженного состояния трубопроводов в режиме испытаний и эксплуатации // Нефть и газ Западной Сибири. Проблема добычи и транспортировки : тезисы докладов Всесоюзной конференции. - Тюмень, 1985. - С. 189-190.

13. Большаков В. Н., Горбаш В. Г., Оленович Т. В. Влияние механических напряжений на локальную остаточную намагниченность // Изв. АН БССР, сер. физ. тех. наук. -1980 . - № 1 . - C. 109-112.

14. Resistance of residual magnetization of heat-treated steel products to elastic deformations / E. S. Gorkunov, V. F. Novikov, A. P. Nichipuruk, V. V. Nassonov, A. V. Kadrov, I. N. Tatlybaeva // Soviet Journal of Nondestructive Testing - USSR. - 1991. - Vol. 27, no. 2. - P. 138-145.

15. Kuleev V. G., Bida G. V., Atangulova L. A. Feasibility of NDT of ferromagnetic steel structures based on measurements of residual magnetization as a function of elastic stress // Russian Journal of Nondestructive Testing. - 2000. - Vol. 36, iss. 12. - P. 866-876. - DOI: 10.1023/A:1016714209905.

16. Novikov V. F., Yatsenko T. A., Bakharev M. S. Coercive force of low-carbon steels as a function of uniaxial stress. Part I // Russian Journal of Nondestructive Testing. - 2001. - Vol. 37, iss. 11. - P. 799-804. - DOI: 10.1023/A:1015899320754.

17. Bida G. V. Magnetic method for estimating uniaxial compressive and tensile elastic stresses // Russian Journal of Nondestructive Testing. - 2011. - Vol. 47, iss. 8. - P. 551-560. - DOI: 10.1134/S1061830911080043.

18. Irreversible changes in the magnetization as indicators of stressed-strained state of ferromagnetic objects / V. N. Kostin, T. P. Tsar'kova, V. E. Loskutov, K. V. Kostin, A. P. Nichipuruk, V. V. Lopatin // Russian Journal of Nondestructive Testing. - 2009. - Vol. 45, iss. 11. P. 786-796. - DOI: 10.1134/S1061830909110059.

19. Measurement of the hysteresis characteristics of pipe steels under elastic and plastic tensile strain / K. V. Kostin, T. P. Tsar'kova, A. P. Nichipuruk, Ya. G. Smorodinskii // Russian Journal of Nondestructive Testing. - 2011. - Vol. 47, iss. 9. - P. 593-602. - DOI: 10.1134/S1061830911090051.

20. Influence of Uniaxial Tension on Magnetic Characteristics of the 12 Gamma(sic) Pipe Steel Exposed to Hydrogen Sulfide / E. S. Gorkunov, S. M. Zadvorkin, I. N. Veselov, S. Yu. Mitropol'skaya, D. I. Vichuzhanin // Russian Journal of Nondestructive Testing. - 2008. Vol. 44, iss. 8. - P. 566-573. - DOI: 10.1134/S1061830908080093.

21. Gorkunov E. S., Zadvorkin S. M., Putilova E. A. Magnetic estimation of stresses applied to a two-layer steel C(T)3-steel 08X18H10T composite material during elastoplastic deformation by uniaxial tension // Russian Journal of Nondestructive Testing. - 2012. - Vol. 48, iss. 8. P. 495-504. - DOI: 10.1134/S1061830912080050.

22. Studies on stress-magnetism coupling effect for 35 steel components / Shang-kun Ren, Yang-chun Ou, Ren-zhen Fu, Yue-wen Fu // Insight: Non-Destructive Testing \& Condition Monitoring. - 2010. - Vol. 52, no. 6. - P. 305-309. - DOI: http://dx.doi.org/10.1784/insi.2010.52.6.305.

23. Possibilities of magnetic inspection of plastic deformations preceding failures of low-carbon steels constructions / A. P. Nichipuruk, A. N. Stashkov, V. N. Kostin, M. K. Korkh // Russian Journal of Nondestructive Testing. - 2009. - Vol. 45, iss. 9. - P. 616-622. DOI: $10.1134 / \mathrm{S} 1061830909090034$.

24. Effect of Elasto-Plastic Loading on the Magnetic Characteristics of Steel 20 Hardened with Gas Case-Hardening / E. S. Gorkunov, S. Yu. Mitropolskaya, E. M. Groznaya, A. N. Mushnikov, A. L. Osintseva, E. A. Tueva // Russian Journal of Nondestructive Testing. - 2011. - Vol. 47, iss. 4. - P. 221-231. - DOI: 10.1134/S106183091104005X.

25. Дунаев Ф. Е. Процессы перемагничивания ферромагнетиков: учебн. пособие. Свердловск : УрГУ, 1979. - 89 с.

Mushnikov A. N. et al. / Effect of mechanical stresses on the magnetic characteristics of pipeline steels of different classes 
26. Effect of mechanical stresses on the magnetic characteristics of pipe steel / E. S. Gorkunov, S. M. Zadvorkin, A. N. Mushnikov, S. V. Smirnov, E. I. Yakushenko // Journal of Applied Mechanics and Technical Physics. - 2014. - Vol. 55, iss. 3. - P. 530-538. - DOI: 10.1134/S002189441403016X.

27. Sablik M. J., Jiles D. C. Modeling the Effects of Torsional Stress on Hysteretic Magnetization // IEEE Transactions on Magnetics. - 1999. - Vol. 35, no. 1, part 2. - P. 498-504. DOI: $10.1109 / 20.737472$. 